\title{
Variations
}

Variations

Revue internationale de théorie critique

$21 \mid 2018$

L'industrie de la culture : version originale

\section{La critique : entre « danger sociologique » et "quiétude négativiste »}

\section{Fabien Granjon}

\section{(2) OpenEdition}

Journals

Édition électronique

URL : http://journals.openedition.org/variations/917

DOI : 10.4000/variations.917

ISSN : 1968-3960

Éditeur

Les amis de Variations

Référence électronique

Fabien Granjon, «La critique : entre « danger sociologique » et « quiétude négativiste » », Variations [En ligne], 21 | 2018, mis en ligne le 05 avril 2018, consulté le 20 avril 2019. URL : http:// journals.openedition.org/variations/917 ; DOI : 10.4000/variations.917

Ce document a été généré automatiquement le 20 avril 2019

Les ami•e•s de Variations 


\title{
La critique : entre « danger sociologique » et « quiétude négativiste "
}

\author{
Fabien Granjon
}

1 Durant les décennies 1980 et 90, la critique avait relativement peu de place dans l'Université française; ses champs connexes, médiatiques et éditoriaux, n'étaient guère plus généreux avec elle. Il a fallu attendre le tournant du siècle et notamment le développement $\mathrm{du}$ mouvement altermondialiste pour voir se revigorer les milieux militants, la critique sociale, et, en écho, la sphère universitaire et les éditions critiques. Nous avons ainsi assisté à une revivification de la critique via la multiplication de séminaires, de colloques, de revues, d'ouvrages, de diplômes, etc. Nous sommes passés, en quelques années, d'une période de disette à une ère de profusion, voire à une production inflationniste en certains domaines. L'hypothèse qu'il semble aujourd'hui raisonnable de poser est que cet élan critique est en train de subir de multiples attaques. Cette situation s'avère, nous semble-t-il, liée à un raffermissement droitier du champ politique et des espaces publics. En vrac: l'importance prise par La Manif Pour Tous contre le mariage gay, la multiplication des espaces de débats entièrement tenus par des experts et des éditorialistes de sensibilité libérale, la dédiabolisation du Front National, la mort des partis sociaux-démocrates, le développement de la fachosphère et du confusionnisme, la montée de la xénophobie et des replis identitaires, etc., sont autant de faits certes distincts, mais qui, pris dans leur ensemble et leur concomitance, concourent à façonner un esprit du temps assez clairement réactionnaire.

2 Sur un plan strictement politique, de nombreux signes tendent à prouver que la période ouvre sur une époque de crise permanente (institutionnelle, idéologique, de légitimité), au sein de laquelle le niveau d'affrontement social pourrait devenir particulièrement élevé et épouser des formes fort variées. La catastrophe écologique imminente et son lien direct avec la question de la justice sociale, le combat contre le pouvoir des banques, des grands groupes et des institutions internationales qui avait animé le mouvement altermondialiste restent un puissant vecteur de « radicalisation » politique, tant dans les 
vieilles démocraties occidentales qu'au sein des pays émergents. Toutefois, ce déploiement conséquent de la conflictualité sociale crée aussi un raffermissement des réponses des gouvernements allant des révolutions passives d'aménagement des politiques de welfare là où elles existent encore, à la libéralisation accrue des services publics et des communs, en passant par le recours à des formes de répression et d'offensives qui fragilisent les démocraties, notamment européennes : mise à mal de certains droits fondamentaux, état d'urgence permanent, sécessionnisme (Brexit), replis identitaires, populistes et ultranationalistes (montée des extrêmes droites en Autriche, Hongrie, Pays-Bas, etc.), émergence des radicalismes religieux (salafisme, islamisme djihadiste notamment), etc.

3 À la faveur de ce contexte, on constate donc un raffermissement évident des politiques et des discours réactionnaires qui s'accompagne d'une volonté pratique de faire taire la critique sociale via des processus de criminalisation des mouvements sociaux, d'accroissement du contrôle social, de matraquage idéologique et de décrédibilisation des analyses, discours et pratiques progressistes. Cette situation de crise a notamment, comme le souligne Bernard Lahire, produit des effets de décomplexion chez certains auteurs qui n'hésitent plus à partager leur détestation de la critique tant théorique que pratique. L'un des fronts spécifiques de cette guerre s'exprime clairement dans les attaques portées aux sciences sociales, tout particulièrement quand elles se revendiquent critiques. Autrement dit, la critique serait soumise, aujourd'hui, à des intimidations d'ampleur et de natures assez différentes, mais visant à amoindrir le rôle qu'elles seraient susceptibles de jouer au côté des forces qui tendent à remettre en cause les ordres sociaux.

\section{Dégagisme et censure}

Pour décrire ce phénomène, nous serions tenté de parler de dégagisme. Par là, nous entendons qualifier les prétentions de certains à vouloir décrédibiliser les sciences sociales, voire tout bonnement les éliminer de la sphère académique, au motif qu'elles ne serviraient pas suffisamment l'innovation et la compétitivité. 26 des 86 universités japonaises ont, en juin dernier (2017), été mises en demeure, par le ministre de l'éducation Hakubun Shimomura, de se débarrasser de leurs départements de SHS. Dans la lettre de mission ministérielle il était ainsi demandé aux universités japonaises de favoriser les disciplines servant de façon plus évidente les «besoins de la société ». Il y a quelques années, en Suisse, Adrian Amstutz, conseiller national bernois du parti UDC, avait demandé que le nombre d'étudiants en sciences sociales soit tout bonnement divisé par deux, arguant que les jeunes feraient mieux de suivre un apprentissage, afin qu'il y ait un vrai retour sur investissement pour la société. Ces propositions utilitaristes et antiintellectualistes aimeraient bien passer les universités sous la tutelle du secteur entrepreneurial ou, à tout le moins, réserver l'exercice des SHS aux universités dites «d'excellence ». C'est assez clairement le modèle américain qui fait ici référence : un enseignement supérieur à deux vitesses. D'un côté des collèges universitaires qui auront pour mission de former la main d'œuvre des bassins d'emploi où ils se trouvent implantés et, de l'autre, des universités prestigieuses, pouvant se permettre de continuer à valoriser la recherche, mais dont les frais d'inscription seront mirifiques. Dans un futur proche? Non, nous y sommes déjà. Il existe par exemple, depuis une quinzaine d'années, une étrange Ligue européenne des universités de recherche qui se définit comme une 
association regroupant des universités de recherche intensive dispensant des enseignements de « haute qualité » et correspondant à un environnement international compétitif. Mais une des formes pratiques et radicales de ce dégagisme s'incarne dans les phénomènes de censure. Ces derniers mois, en France, les tentatives de censure des sciences sociales et de la connaissance critique se sont en effet multipliées.

Citons-en quelques exemples, toujours sur le mode du vrac. Il y a eu, par exemple, le déclassement, à un concours CNRS, de jeunes sociologues aux bénéfices de juristes, alors qu'ils étaient pourtant classés premiers par le jury d'admissibilité. Leurs noms ont même disparu de la liste complémentaire dressée par le jury d'admission. Une première. Le carnet de recherche RussEurope de Jacques Sapir, hébergé sur la plateforme Hypothèses d'OpenEdition a été fermé au motif que l'auteur y aurait publié des textes partisans déconnectés du contexte académique. Citons aussi le cas de cette interdiction décrétée puis retirée - par l'Université de Strasbourg concernant l'expression publique de ses enseignants-chercheurs ou celui de l'Université d'Avignon et des pays de Vaucluse, laquelle s'est distinguée en intentant un procès ubuesque à l'un de ses enseignants au motif de " provocation à la discrimination raciale » et ce, pour un courriel envoyé sur une liste de débat interne à l'université à l'occasion de la venue de Manuel Valls ; e-mail qui reprenait ironiquement certains propos sur les « blancos » tenus à une autre occasion par le Premier ministre. Mentionnons également les polémiques violentes et les intimidations contre les initiatrices du camp d'été décolonial non-mixte de formation à l'antiracisme politique, celles dirigées contre le collectif Mwasi qui organise Nyansapo, un festival afroféministe, ou encore celles qui ont voulu empêcher la tenue d'un colloque sur l'intersectionnalité dans les recherches en éducation, lequel s'est finalement tenu à l'ESPE de Créteil. Et puis il y a eu quelques interdictions effectives : annulation d'un colloque sur l'islamophobie à l'Université Lyon 2, pourtant organisé par la Chaire Égalité, Inégalités et Discriminations, au motif de la participation à l'événement de militants luttant contre... les discriminations ; interdiction de séminaires à l'Université Rennes 2 et à l'Université de Limoges auxquels était invitée la porte parole du PIR, Houria Bouteldja; déprogrammation par ScePo Paris d'une conférence sur les rapports entre le régime russe et les activités terroristes; interruption violente d'une conférence d'Édouard Louis et Geoffroy de Lagasnerie à l'Université de Lille 3, etc. On peut également citer le cas de ce droit de réponse diffamatoire rédigé par un maire mécontent de l'analyse rédigée par une professeure de droit dans le revue AJDA, concernant un jugement du tribunal administratif qui annulait la délibération de son conseil municipal prévoyant la suppression des menus de substitution dans les cantines de sa commune. Et la liste n'est évidemment pas exhaustive. Une censure plus insidieuse mais tout aussi efficace tient évidemment à la réduction des budgets de recherche, les laboratoires étant de plus en plus sommés de trouver des financements à l'extérieur de l'espace académique et notamment au sein de la sphère entrepreneuriale. Pour des recherches critiques, la possibilité de décrocher des subsides conséquents, hors champ universitaire, est bien sûr assez mince. Valérie Pécresse, Présidente de la région Île-de-France, a ainsi officialisé le fait que ladite région ne financerait plus les recherches sur le genre, les inégalités et les discriminations et qu'elle retirait donc ces thématiques de ses "Domaines d'intérêt majeur ». 


\section{Danger sociologique et coup médiatique}

6 Outre ces velléités pures et simples d'élimination, il existe des formes de dégagisme plus ciblées en ce qu'elles proposent de se débarrasser plus particulièrement des sciences sociales les plus critiques. Le livre de Gérald Bronner et Étienne Géhin, Le danger sociologique (PUF, 2017) ou le numéro de novembre-décembre 2017 de la revue Le Débat, sont les derniers avatars en date de ce type d'avanie. Bronner et Géhin parlent à cet égard de "sciences sociales déterministes", histoire de faire passer l'importance donnée aux déterminations sociales comme une vulgaire attention mécaniste. Ils se réfèrent à Raymond Boudon et s'appuient sur le paradigme de l'individualisme méthodologique pour avancer leurs thèses. Sur le fond, leur ouvrage n'est pas franchement innovant puisqu'il se contente de rejouer les batailles intestines qui ont émaillé les sciences sociales dés leurs premiers développements. Le livre relance, en effet, les controverses qui concernent le grand partage entre l'explication et la compréhension, entre l'objectif et le subjectif, entre le nomothétique et l'herméneutique, entre le holisme méthodologique et l'individualisme méthodologique, entre les méthodes des sciences de la nature et celles des sciences de l'esprit, entre le positivisme et le culturalisme, entre le déterminisme et la liberté, etc. Force est de constater que ces débats, bien qu'anciens, continuent à traverser le champ de production scientifique et, à des fréquences régulières, donnent lieu à des controverses importantes, à l'instar des échanges bien connus entre Karl Popper et Theodor W. Adorno ou ceux, plus récents, entre la sociologie critique bourdieusienne et la sociologie de la critique boltanskienne. Si la chose n'est donc pas nouvelle, il est toutefois intéressant de s'interroger sur le fait que le pamphlet de ces deux collègues a bénéficié d'une visibilité et d'une publicité très importantes, dont on peut faire l'hypothèse qu'elles ne sont pas seulement la conséquence d'un marketing éditorial efficient, mais qu'elles résonnent aussi avec une demande sociale propre à la période.

7 Un article d'Acrimed (http://www.acrimed.org/Le-Point-et-Pour-la-science-mettent-del-ordre?recherche=bronner) pointe en effet l'attention toute particulière qui a été apportée à cet ouvrage, y compris sur les antennes de la radio publique et de l'opportunité que cela a représenté, pour un ensemble de journalistes, de dire leur détestation de la sociologie. Lahire note que ce sont les médias qui ont organisé la sortie de cet ouvrage comme un événement, donné un peu partout la parole à Bronner et caricaturé la sociologie critique comme une simple collection d'opinions politiques, c'està-dire, dans leur esprit, non-scientifiques. L'un des arguments centraux de Bronner et Géhin est précisément d'avancer que les sciences sociales doivent se départir de leur gangue idéologique pour recouvrer une scientificité digne des sciences de la nature, de la cumulativité et du principe d'une stricte réfutabilité. Le refrain est d'ailleurs entonné par nombre d'autres confrères tel Jean-Louis Fabiani qui dans l'émission de France Culture $D u$ grain à moudre évoque, par exemple, les velléités " prophétiques » et le "pathos social » de Pierre Bourdieu. Dans le récent ouvrage (Seuil, 2016) qu'il lui consacre, sous-titré « un structuralisme héroïque », Bourdieu est notamment dépeint, à la fin de sa vie, comme obsédé par une réflexivité autocentrée et la nécessité de montrer qu'il aurait été l'initiateur d'une révolution symbolique majeure. $\mathrm{Si}$, en l'espèce, il ne s'agit pas encore tout à fait de faire passer la critique pour une fantaisie romantique, Bronner et Géhin, eux, n'hésitent pas à faire le pas et à considérer la critique comme un héroïsme réservé aux indignés qui se voudraient davantage du côté du bien que du vrai. On connaît la 
réfutation que Bourdieu apporte à cet argument qui ne date pas d'aujourd'hui, tant s'en faut. La dichotomie entre scholarship et commitment est, selon lui, inepte dans la mesure où il faut être un savant respectueux des règles du scholarship pour pouvoir produire un savoir engagé, c'est-à-dire un scholarship with commitment. Cette opposition permettrait juste à certains chercheurs de se croire doublement savants, parce qu'ils ne font rien de leur science ajoutait-il.

\section{Les neurosciences à la rescousse}

C'est pourtant au nom de cette séparation factice et stérile que Bronner et Géhin entendent donner une place importante aux sciences cognitives, à la neurobiologie. De l'IRM comme appareil de preuves! Ils estiment que les neurosciences démontrent que le cerveau fait fatalement de l'individu un sujet social inventif et doté d'un puissant librearbitre. On pourra, à cet égard, s'étonner de porter au pinacle la neurobiologie et l'individualisme libéral tout en dénonçant le supposé déterminisme de la sociologie critique et en comprenant bien qu'il s'agit là d'un passage obligé pour réinscrire dans le domaine du «naturel » ce qui précisément est aussi toujours une construction sociale. Cette référence aux sciences cognitives est, elle aussi, bien dans l'esprit du temps. Le prix Nobel d'économie Richard Thaler, n'est autre que la tête de pont du courant Behavioral Economics. Les neurosciences sont aussi de plus en plus présentes en France, par exemple dans des politiques gouvernementales. Le Ministre de l'éducation nationale, Jean-Michel Blanquer a ainsi récemment mis en place un conseil scientifique de l'éducation nationale dont l'objet est de développer une pédagogie qui, enfin, serait fondée sur de réelles preuves scientifiques. À la tête de cette instance de consultation se trouve un professeur de psychologie cognitive expérimentale du Collège de France, Stanislas Dehaene, qui estime que l'enseignement est une science et affirme que ce qui le motive, c'est d'agir indépendamment de toute idéologie.

On l'aura compris, il s'agit donc d'opposer la science à l'idéologie, la sociologie analytique à la sociologie critique et, depuis ce grand partage, ripoliner les vieilles lune de la neutralité axiologique, de la suspension des jugements de valeur, et revigorer la détestation du principe de totalisation considéré par les sots comme une forme de généralisation abusive. Bronner n'a d'ailleurs de cesse de justifier son retour à un positivisme naïf au nom de la complexité d'un réel qui pourtant pourrait être finalement ramené à l'individu, ses comportements et ses calculs. Il y a là comme une évidente aporie. Or opposer les sciences cognitives aux sciences sociales au nom de leur plus grande scientificité, c'est s'interdire de penser que les capacités cognitives sont évidemment aussi le produit de la socialisation et indissociables des formes de vie sociale. Dans un ouvrage codirigé avec Claude Rosenthal (EAC, 2008), Lahire souligne à raison que le rôle des sciences sociales est d'analyser la formation sociale et la variation des structures mentales-comportementales, et d'ancrer dans des configurations historiques ce qui apparaît aux sciences cognitives comme des réalités universelles. La contextualisation par la prise en compte à parts égales de l'histoire et des relations d'interdépendances à différents niveaux d'échelle se présente comme le travail de base des sciences sociales les plus sérieuses. Historiciser des états de fait, dénaturaliser et défétichiser les évidences, désessentialiser les différencialismes aveugles aux milieux, penser les évolutions et les transformations sociales réelles... Il ne s'agit pas de faire autre chose que cela et c'est évidemment énorme. Or c'est au nom de la scientificité et contre l'idée d'une science normativement fondée 
que Bronner et Géhin s'opposent justement à la prise en compte des déterminations sociales et des dynamiques collectives qu'ils considèrent comme des instances métaphysiques sans existence réelle, et ce, au profit de ce qui se passerait «dans la tête des acteurs » et au nom de l'imprédictibilité fondamentale des faits mentaux et sociaux. Parallèlement à ce qu'ils nomment le « biais d'agentivité »- syntagme qui, sous leur plume, désigne une critique de la supposée fictionnalisation de la réalité sociale par invention d'instances collectives et de structures prescriptives -, ils récusent l'idée que toute production scientifique est le précipité d'un regard qui est toujours le fruit d'un point de vue qu'il s'agit d'objectiver.

10 La sociologie analytique serait donc neutre car scientifique, exempte de tout point de vue. Pourtant, Bronner est bien connu pour ces accointances avec la grande industrie et LREM, ce qui lui vaut, sous la plume toujours acérée de Frédéric Lordon, le sobriquet d'« ambianceur sociologique du macronisme et de la presse de droite ». Sans doute s'agitil de considérer cette orientation comme une forme de neutralité engagée, comme le défendrait certainement la sémillante Nathalie Heinich. Bronner et ses comparses lancent là une énième tentative de dépolitisation et de désarmement du savoir sociologique que d'aucuns aimeraient bien éloigner de toute velléité à prendre (sa) part aux processus de transformation de la société. C'est au principe de ce genre de considérations que Bourdieu est devenu ce «mandarin rouge » haïssable, à partir du moment où, au mitan des années 1990, il a pris fait et cause pour le mouvement social et reconnu plus explicitement qu'auparavant, le lien existant entre le savant et le politique. Une des manières de se débarrasser de vérités gênantes, affirmait justement Bourdieu, est d'affirmer qu'elles ne sont pas scientifiques, mais politiques. Bronner et Géhin voudraient donc faire le ménage et reprennent à cet effet la ritournelle de la supposée nécessaire désextrémisation et décomplotisation de la pensée sociologique qui, dans une perspective critique, aurait notamment le tort, depuis ce qu'ils considèrent comme un "populisme de la résistance ", d'essayer de saisir les phénomènes de domination. Comme le rappelle Lahire, les dominations et les inégalités sont néanmoins des faits qui s'observent, se mesurent, s'objectivent et ne sont pas seulement des constructions théoriques hors sol. Le problème que pose la critique à Bronner et Géhin est qu'elle dénaturalise, démystifie, qu'elle défétichise et par là, dérange les intérêts de ceux qui ont précisément intérêt à maintenir sous cape un certain nombre de faits et en particulier ceux qui tendraient à expliciter le caractère construit de leurs positions dominantes, si ce n'est en certains cas de leurs privilèges. Dans les pages du Point, Alain Touraine affirme ainsi que la sociologie critique ne serait « qu'une sorte de pseudo-marxisme qui réduit tout à l'inégalité ».

\section{Déresponsabilisante et performative}

11 Cette argumentation rentre par ailleurs en résonnance forte avec les propos tenus par certains médiacrates et hommes politiques quant au «sociologisme » et à la « culture de l'excuse » qui traverseraient les sciences sociales. Dans cette variante, le dégagisme que nous évoquions est justifié par les supposés discours de déresponsabilisation que tiendraient les sciences sociales. On a pu lire ce genre de niaiseries dans le livre de Philipe Val, ex-éditorialiste de Charlie Hebdo et islamophobe convaincu: Malaise dans l'inculture (Grasset, 2015), dont le titre détourne celui du célèbre ouvrage de Freud et dans lequel il dénonce le sociologisme qui excuse. Dans un entretien donné à L'Express, il affirme qu'une certaine sociologie nie la responsabilité et la liberté de l'individu. Il ajoute que quand on 
en fait une idéologie, ça provoque des millions de morts... Lahire a courageusement consacré une quarantaine de pages de Pour la sociologie. Et pour en finir avec une prétendu «culture de l'excuse » (La Découverte, 2016) à ce tissu d'imbécilités qui fait de la sociologie le nouvel avatar d'une vision que Val qualifie de «stalino-marxiste». Il faut se pincer pour y croire. Un livre d'époque conclut Lahire. De son côté, Manuel Valls, quand il était Premier ministre, affirmera, à propos d'analyses tenus par des chercheurs en sciences sociales sur les phénomènes de radicalisation - expliquant que les terroristes sont, qu'on le veuille ou non, le produit de la société au sein de laquelle ils ont vécu -, en avoir marre de « ceux qui cherchent en permanence des excuses ou des explications culturelles ou sociologiques à ce qui s'est passé » (i.e. aux attentats du 13 novembre 2015). Pour l'ancien séide du libéralisme socialiste, «il ne peut y avoir aucune explication qui vaille. Car expliquer, c'est déjà vouloir un peu excuser ». Si, en ce cas d'espèce, la saillie vallsienne paraît à tout le moins outrancière, des raisonnements similaires se développent au sein même du monde des sociologues, comme par exemple sous la plume de Thierry Tirbois, enseignant à Paris-Sorbonne, qui écrit sur le blog nonfiction.fr qu'à force de dire aux enfants de milieux modestes qu'ils ne peuvent y arriver, ils finissent par le croire ! (https://www.nonfiction.fr/article-9089-la-sociologie-est-une-science-humaine-pas-unsport-de-combat.htm).

Dans leur contribution au numéro de novembre-décembre 2017 de Le Débat, Bronner et Géhin enfoncent le clou des supposés savoirs-croyances produits par la critique. Ceux-ci dévitaliseraient les notions de mérite, de responsabilité ou de moralité et conduiraient à une "culture de l'excuse». La critique serait donc complice d'un «biais d'autocomplaisance » et les chercheurs critiques devraient réfléchir à deux fois avant de mettre en avant la force des déterminations sociales, car l'exercice pourrait conduire à une forme d'advocacy research qui produirait ce qu'elle cherche en le cherchant! L'accusation consistant à dénoncer le discours sociologique critique comme performatif pourrait prêter à rire s'il n'était pas si répandu, y compris parfois jusque dans les rangs de chercheurs revendiquant une approche critique. On peut par exemple lire ce genre d'inanités dans un livre de Danielle Tartakowsky, ex-Présidente de l'Université Paris 8 et historienne marxiste spécialiste de la manifestation qui, en son temps, avait notamment contribué à L'anthologie Communication and Class Struggle dirigée par Armand Mattelart et Seth Siegelaub (IG/IMMRC, 1979 et 1983). Son ouvrage intitulé Construire l'Université au XXI e siècle (Éditions du détour, 2017) dresse le bilan de sa mandature à la tête de l'institution dionysienne. Elle y dénonce, sans ciller, l'excellent ouvrage de Nicolas Jounin, Voyage de classe (La Découverte, 2014), livre de sociologie critique qui «instrumentaliserait» Bourdieu (sic) et démontrerait aux étudiants qu'ils sont sociologiquement condamnés à demeurer des exclus. Elle affirme préférer Eloquentia, initiative populiste qui propose à ces mêmes étudiants un programme d'expression publique et d'éloquence qui, selon ces termes, permettrait de combattre les obstacles psychologiques et les réflexes d'autocensure qui paralyseraient les jeunes qui auraient intériorisé les stigmates dont les médias ou d'autres les affublent. Ou quand les bateleurs en tous genres sont distingués et les enseignants-chercheurs scrupuleux et innovants dénigrés...

Bronner et Géhin avancent que certains discours sociologiques critiques diffusés dans l'espace public peuvent représenter un danger. Lahire a largement répondu à cet argument dans Pour la sociologie, ainsi que dans quelques émissions radio et articles ${ }^{1}$. Invité par Caroline Broué dans son émission La Grande table (https://www.youtube.com/ watch?v=Abhsd_6VtmA), il a rappelé que derrière les attaques visant le «sociologisme ", 
c'est-à-dire une supposée dérive des analyses sociologiques, ce sont bien les sciences sociales en tant que telles et encore davantage leurs développements critiques qui sont visés et décriés. L'accusation, reprise lors de cette émission par l'essayiste Brice Couturier, tient à ce que la sociologie, en tâchant d'expliquer et de comprendre exonèrerait les individus et en ferait des victimes plutôt que des coupables, en renvoyant toutes les causes des actions qu'ils peuvent commettre du côté de la société et des déterminations liées aux milieux sociaux, notamment aux détriments des vecteurs idéologiques et culturelles. Lahire a eu raison de rappeler qu'il s'agissait là d'une erreur grossière de découpage. La culture, l'idéologie, la religion, la communication, le politique, etc., ne sont pas des domaines autonomes qui auraient une réalité propre, en dehors du social. Le prétendre c'est ne rien y comprendre, tout comme de considérer qu'expliquer ce qui rend probable et possible les faits sociaux, ce serait les cautionner. En revanche, les détracteurs sagaces des sciences sociales et de la critique ont très bien perçu le rôle que celles-ci peuvent jouer dans la mise au jour des logiques de domination à l'œuvre et des visions conservatrices qui les justifient par des discours mâtinant naturalisme et philosophie de la responsabilité individuelle. Pour donner un exemple simple, Lahire évoque les pauvres qui, dans cette perspective, sont pauvres parce qu'ils ne se sont pas donnés les moyens de s'arracher à leur condition et les riches qui seraient riches du fait des efforts qu'ils auraient consenti à faire pour devenir riches. Nous ajouterons que cette vision simpliste de la réalité sociale évite surtout tout effort dialectique permettant de montrer non seulement que la distribution inégale des richesses n'a pas grand chose à voir avec des degrés de volonté et de responsabilité différenciés, mais, surtout, empêche de penser que la richesse des uns peut être la cause de la pauvreté des autres.

\section{Éloigner la sociologie du politique}

14 On l'aura compris, l'idée sur laquelle fait fond ce battage tient à ce que la sociologie critique serait par trop politique, notamment quand elle s'attaquerait à montrer en quoi les inégalités et les formes de domination sont produites et reproduites par des institutions, des structures, des systèmes qui n'ont rien de naturels et qu'il s'agit de ne pas méconnaître si l'on veut les combattre. Ce but politique de la critique qui vise à suspendre l'adhésion préréflexive au monde tel qu'il va et qui veut se mêler à sa manière et depuis ce qu'elle est de la praxis sociale tend à être fortement décriée. L'idée que puisse par exemple se constituer des lieux de débats - journées d'études, colloques, etc. -, mélangeant milieux universitaires et milieux militants est aujourd'hui ouvertement critiquée. Si l'on comprend, pour ne prendre que cet exemple, que les propos d'Houria Bouteldja sur le philosémitisme, le viol, l'homosexualité ou encore la "blanchité » puissent être perçus comme pour le moins discutables, il faut donc les discuter. La tribune sortie dans Le Monde du 30 novembre 2017 à l'initiative du sociologue Alain Policar et de l'historien Emmanuel Debono qui s'indignent, avec d'autres, de «la multiplication des colloques à visées militantes » ne nous semble pas poser le problème correctement car elle fait fond sur la nécessité de devoir séparer recherche et communautés politiques organisées. S'il semble possible de suivre ces collègues sur le fait que l'antiracisme politique de Bouteldja fleurte, quoi qu'en disent ces fébriles soutiens, avec une pensée essentialiste à tendance racialisante, il est nettement plus douteux de devoir accepter le fait que, comme ils l'affirment, la problématique du racisme ne serait pas nécessairement nourrie par « l'ethnocentrisme, le capitalisme et les survivances du colonialisme ». 
15 À cet égard le $n^{\circ} 197$ de la revue Le Débat s'avère très exactement du même acabit que le Danger sociologique : la réplique d'un séisme de faible amplitude faisant cependant la une des médias. Dominique Schnapper s'y inquiète évidemment de la politisation de la recherche, qui serait, selon elle, la grande tentation des sociologues français qui viseraient à remplacer la discussion rationnelle par des dénonciations de nature politique. On en déduit donc que la production de connaissances engagées serait nécessairement une opération éloignée de la rationalité. C'est, ce qu'il y a quelques années, faisait avancer à Nathalie Heinich commentant l'ouvrage de Christine Delphy, Classer, dominer. Qui sont les autres (La Fabrique, 2008) qu'aucun des textes du recueil en question n'aurait pu trouver place dans une revue scientifique, " même de bas niveau » ajoutait-elle, et que Delphy usurpait son poste de chercheuse au CNRS. Si la sociologie ne saurait être, pour Heinich et quelques autres, un sport de combat, elle pourrait, semble-til, sonner l'ouverture de la chasse aux sorcières. Pour sa part, Pierre-Michel Menger, estime que la critique dénonce davantage qu'elle analyse et démontre. Il oppose deux ontologies : une première, critique, qui n'aurait vocation qu'à inscrire les faits sociaux dans des systèmes de contraintes, rabattrait toute explication sur le consentement inconscient à l'arbitraire des rapports de force et aurait, par conséquent, quelque difficulté à penser le mouvement, l'innovation le changement. A contrario, une seconde ontologie, non critique, donnerait tout «crédit à la qualification processuelle de la réalité » contre " la menace fataliste de l'éternel retour du même ». On trouve également, dans cette livraison hivernale, Nathalie Heinich, fidèle au poste d'arbitre des élégances sociologiques. Elle nous livre un « Misère de la sociologie critique » qui étale une nouvelle fois, sans surprise, sa détestation des sciences sociales engagées. À son habitude, elle considère les approches critiques comme relevant de la préhistoire de la discipline et rejette la normativité dans le préscientifique. Pour l'ex-étudiante de Bourdieu, la science ne peut bien sûr avoir partie liée avec le politique et le constructivisme critique qui, selon Marcel Gauchet, serait un « inexistentialisme ». Gauchet qui, au passage, estime, lui aussi, que les sciences sociales ne nous apporteraient plus grand chose dans la mesure où elles se seraient rendues incapables de saisir ce qui se passe dans la tête des gens. Pour Heinich, la critique serait doxique et dogmatique quelles qu'en soient les versions - École de Francfort, sociologie de la domination, études de genre, etc. - et interdirait que soient énoncées certaines vérités. La proscription du vrai est pourtant précisément ce qu'elle préconise en estimant, par exemple, que l'antisexisme devrait rester cantonné à la sphère militante, voire en suggérant que la sociologie pourrait, en l'espèce, n'avoir aucune vérité scientifique à produire sur la question. Olivier Galland, autre mousquetaire du Débat faisant feu sur la critique estime que ce qu'il nomme la «sociologie du déni » produirait des discours victimaires. Pour Lahire, justement, il s'agit là d'une charge évidente contre toutes celles et ceux qui rappellent les traitements inégaux subis par les différentes catégories de la population, mais c'est aussi un positionnement idéologique qui confère, en l'occurrence, la responsabilité de leur situation aux immigrés eux-mêmes et à leurs descendants. Il voit dans cette livraison une droitisation de la vie intellectuelle qui vilipende sans grand talent les approches critiques qui prennent en considération les ordres sociaux et les phénomènes de domination.

Dans Le Danger sociologique, Bronner et Géhin tiraient déjà à boulets rouges façon « Grosse Bertha » sur le paradigme holistique dont Karl Marx dans sa Critique de l'économie politique avait par exemple donné une illustration dessillante dans son explication des rapports sociaux de production indépendants de la volonté des hommes y prenant part et 
correspondant à un niveau de développement des forces productives matérielles. Pour Marx, l'ensemble de ces rapports forme l'infrastructure économique à laquelle correspondent une superstructure et des formes particulières de conscience sociale. On connaît également les précisions d'Engels dans sa lettre à Joseph Bloch sur le fait que si les conditions économiques sont absolument déterminantes, les conditions politiques et les représentations jouent aussi un rôle non négligeable. Nous effectuons ici cette précision car les attaques portées à la critique sont largement empruntes d'un refus de reconnaître l'effectivité des ordres sociaux, des structures, des classes, etc. Cyril Lemieux, qui ne peut pas être accusé d'être un dangereux sociologue gauchiste, estime à raison, dans un entretien donné à L'Obs (https://bibliobs.nouvelobs.com/ idees/20170929.0BS5360/la-sociologie-est-elle-depassee-le-debat-gerald-bronner-cyrillemieux.html), que les approches holistiques qui refusent de décorréler l'individuel et le collectif, l'un faisant l'histoire de l'autre et réciproquement, heurtent frontalement le raisonnement libéral. Il y voit d'ailleurs l'origine d'un paradoxe dans la mesure où plus la société devient individualiste et s'efforce de ne pas penser sociologiquement les rapports sociaux, plus le besoin de sociologie se fait sentir, mais plus celle-ci apparaît comme honteuse et se voit désactivée dans ses potentialités.

\section{Holisme et totalité}

Dans Socialisme et sociologie (éditions EHESS, 2017), Lemieux et Karsenti soulignent à raison combien l'économie, le droit ou encore la psychologie entretiennent un lien étroit avec le libéralisme, c'est-à-dire avec les disciplines qui privilégient l'individualisme méthodologique. Ils nuancent d'ailleurs le propos en précisant qu'il s'agit, dans ces disciplines, d'une tendance et que les courants internes qui ne s'y conforment pas y sont généralement critiques et minoritaires. Il faudrait voir également pour d'autres sciences sociales autonomisantes, comme par exemple les sciences de l'information et de la communication (SIC) ou les Cultural Studies; effectuer un travail d'enquête précis sur les tendances intellectuelles de ces sous-champs et se donner les moyens de rendre compte de la structuration de ces régions épistémologiques depuis les rapports plus ou moins affinitaires qu'elles entretiennent à l'individualisme méthodologique. Pour ce qui concerne les SIC, la chose nous semblerait tout particulièrement intéressante dans la mesure où elles ont un statut épistémique fort singulier. Elles se présentent comme des sciences sociales particulières en ce qu'elles choisissent d'abstraire une catégorie de phénomènes sociaux à étudier, sauf que cette opération de spécification par la communication les maintient à un étiage qui serait plutôt celui d'une science synthétique. Ce flou des approches dites «communicationnelles » ou la diversité des épistémologies des Studies conduit à des positionnements fort variés quant à la place à octroyer aux explications faisant la part belle, en dernière instance, aux comportements individuels. Ce qui est certain tient à ce que les travaux qui s'y mènent et qui s'appuient sur des espaces d'objectivation qui préfèrent s'intéresser aux substances, essences, identités, plutôt qu'à la formation sociale des faits et des sujets sociaux, sont aussi ceux pour qui le paradigme holistique et l'idée de totalisation chère au matérialisme dialectique sont considérés, à tout le moins au sein des visions les plus outrancières, comme l'antichambre du totalitarisme. C'est d'ailleurs sous cet aspect que Bourdieu a maintes fois été injurié et dépeint en mandarin sectaire, le couteau entre les dents, à la tête d'une secte ou en collusion avec on ne sait quel autre collectif dangereux. C'est également à ce même motif 
que Raymond Boudon, dans son Dictionnaire critique de la sociologie (Paris, PUF, 1982), renvoyait la notice sur le « holisme » à l'entrée sur le « totalitarisme ».

C'est vraisemblablement pour ces mêmes raisons qu'un vague collègue nous a harcelé durant des mois en nous expliquant que la critique matérialiste était le ferment de la politique du goulag, ou qu'un autre, n'arrivant pas à considérer ses pairs Professeurs comme autre chose que des mâles blancs dominants sexistes et malveillants - gênant ses velléités carriéristes -, de considérer qu'une politique scientifique critique conséquente revenait inévitablement à mettre en œuvre un « système de police de la pensée, marxisteléniniste totalitaire ». Pour donner un dernier exemple, la recomposition récente de notre laboratoire en deux équipes a été justifiée par nos collègues schismatiques par la nécessité de donner «toute son importance aux choix individuels de chercheur.e.s » et de « ne pas représenter la société comme un seul "sujet en grand" dominé par des structures économiques et sociales, aussi puissantes soient-elles». On s'étonnera que de telles "puissantes structures" puissent ne pas être prises en compte, mais aussi que de la préséance accordée à la totalité puisse se déduire mécaniquement un manque d'attention accordé aux cadres de pensée, aux subjectivités et aux processus de subjectivation. Être vent debout contre la raison dialectique, le paradigme holistique et le principe de totalisation amène ainsi à proférer quelques assertions pour le moins approximatives. De surcroît, cela ne vaccine évidemment pas contre le fait de nourrir quelque prétention à l'hégémonie théorique et de considérer ses propres modèles d'analyse comme devant être au cœur de la réforme des sciences sociales (la modestie fait rarement bon ménage avec les egos aux volumes "montgolfiers ») dont on souhaite qu'elles se fragmentent en une myriade de Studies qui, enfin, feraient droit à la diversité et l'expressivité du chercheur.e comme de l'enquêté.e.

Dans sa Théorie du sujet (Seuil, 2008), Alain Badiou affirmait que le fait de ne pas céder sur certains éléments théoriques ou éthiques conduisait généralement à se faire accuser de stalinisme. L'effort de totalisation considéré comme antichambre du totalitarisme et/ou comme barrière à penser le complexe, l'imprédictible et l'individu est toutefois une ritournelle bien peu convaincante. Il y a souvent une incompréhension quant à ce à quoi correspond cet effort de totalisation. D'un point de vue épistémologique, le principe de totalité pose que la société est structurée par des contradictions et des rapports sociaux objectifs et subjectifs, qui sont à l'origine de faits variés qui sont le précipité non mécaniste de ces rapports sociaux. Ils en portent donc l'empreinte et ne peuvent ainsi être correctement saisis que s'ils sont pensés au regard de la totalité sociale à laquelle ils prennent part. Aucun fait social, aucun sujet social ne saurait être considéré comme une monade ou une instance strictement individuelle. Lucien Goldmann évoquait, pour sa part, l'existence d'une structure significative, unité du multiple et plaidait pour la mise en œuvre d'un structuralisme génétique attentif à l'histoire et aux structures sociales. JeanMarie Brohm parle, lui, de «complexité synchronique» et de "complexité diachronique ». Tout cela est grosso modo équivalent. Aussi, comprendre la totalité exige également l'analyse des interrelations des différentes parties qui la composent. Par ailleurs, il est entendu que la totalité matérialiste n'est pas quelque chose d'immuable, elle est une totalité en mouvement, produit d'une histoire qui en cadre les déterminations qui sont elles-mêmes en évolution, réorganisant leurs articulations et leurs fonctions. C'est la totalité détotalisée de Sartre, une totalité concrète et contradictoire à considérer comme un ensemble structuré qui ne cesse d'évoluer et doit être appréhendé à l'aune d'une approche historicisante et relationnelle. À sa manière, la sociologie de Bourdieu 
n'est pas éloignée de ce principe, par exemple dans l'interrelation qu'il met au jour entre le champ et l'habitus, l'histoire faite corps et l'histoire faite chose, etc. Luc Boltanski reconnait également que la sociologie peut fournir des schèmes de totalisation qui aident notamment les sujets sociaux à construire un contexte dans lequel ils peuvent se situer. Récuser l'effort de totalisation, c'est tout bonnement s'interdire une pensée robuste des ordres sociaux et prendre le risque de voir dans le moindre bricolage, détournement, décodage ou dans le nombrilisme et l'individualisme, des gestes et des dispositions qui pourraient être révolutionnaires. Dénigrer les approches holistiques, c'est faire comme si l'histoire pouvait être soluble dans le spectacle, le social dans les fluctuations individuelles et la critique radicale - celle qui va à la racine - soluble dans le brouet postmatérialiste.

Pour terminer, reconnaissons qu'il est un point avec lequel nous nous trouvons en accord avec Schnapper, c'est sur le fait que les chercheurs en sciences sociales qui prétendent ne pas céder à l'esprit du temps ne rechignent en revanche pas à s'inscrire dans l'esprit dominant de leur milieu. Il pèse bien évidemment un certain nombre de contraintes sur les pratiques de production des connaissances et de leur diffusion dans la mesure où cet exercice professionnel se déploie dans le cadre d'une institution publique et des tutelles qui nourrissent des attendus singuliers dont le respect assure globalement l'intégration à celles et ceux qui s'y plient. Même (surtout) critique, l'homo academicus est invité à se soumettre à certaines docilités : carriérisme, communautarisme académique, suivisme, etc. Il ne s'agit en aucun cas de donner des leçons, mais il est important d'envisager en quoi l'attachement et l'adhésion aux règles du jeu du champ universitaire et à ses sousespaces peut être un frein à l'exercice d'une recherche critique. L'Université et ses lieux connexes (HCERES, CNU, sociétés savantes, etc.) produisent des recommandations, voire parfois des injonctions à se conformer à des manières d'être et d'agir qui tendent au désarmement d'un savoir engagé. Il y aurait beaucoup à dire sur ce point... Nous voudrions commencer par pointer le fait que la querelle dont on vient de dire deux mots, opposant artificiellement holisme et individualisme, se retrouve également au centre des politiques universitaires qui, globalement, favorisent l'hyperindividualisation des parcours professionnels - laquelle ne fera que prendre de l'ampleur quand l'évaluation des carrières sera mise en place par l'HCERES -, et encouragent le désintérêt pour les collectifs de recherche traditionnels au profit d'une recherche par projet.

Par ailleurs, la disciplinarisation de l'activité de recherche, couplée à l'hyperspécialisation, conduit à une division du travail de production scientifique néfaste à la compréhension globale des dynamiques sociales et des dominations, tout comme à la possibilité de se donner les moyens « d'intervenir sociologiquement en politique » comme le suggère Gérard Mauger. Le réflexe disciplinaire est bien souvent une réaction de défense de positions acquises dans le champ et une forme de réassurance quant au fait d'appartenir à un groupe, d'en être, parfois drapée dans une urgence à défendre une singularité qui ne résiste pourtant pas toujours à l'examen. Ces complications institutionnelles semblent d'ailleurs faire bon ménage avec le narcissisme fractionniste des petites différences critiques qui cultive les dissemblances et les nuances davantage par distinction que par réelles nécessités heuristiques et pratiques. De la même façon que certaines «nouvelles » radicalités " politiques semblent principalement occupées à haïr et liquider toutes les gauches - Carthago delenda est -, certains développements critiques débouchent sur ce que Walter Benjamin nomme une quiétude négativiste - «se situer à la gauche de tout possible en général » écrit-il -, qui s'emploie davantage à envisager les 
autres positionnements critiques comme des obstacles aux tentatives de construction de leur hégémonie, qu'à considérer ceux-ci comme de potentiels partenaires avec qui ils pourraient être utiles de discuter autrement que par des fulminations, des anathèmes et des décrets de supériorité. L'idée d'un rapprochement possible, d'un commun, d'un dépassement des contradictions est jetée aux oubliettes avec la dialectique, l'universalisme, la possibilité de politiques communes et de convergences des luttes, en théorie comme en pratique. Il existe, hélas, une critique autophage - pour parler comme Jacques Bouveresse - et onaniste qui, dans certains de ses jouissifs développements (nihilisme, cynisme, anti-progressisme, etc.) n'est pas si éloignée que cela de certains positionnements parmi les plus réactionnaires.

Enfin, l'attentisme et le suivisme de la plupart des universitaires, y compris critiques, visà-vis des politiques libérales de casse du service public d'enseignement supérieur (sélection, évaluation, excellence, regroupement des établissements, bureaucratisation, publish or perish, concurrence, course à l'indice de Hirsch, etc.) contribuent à faire de l' alma mater un espace de plus en plus incompatible avec les exigences d'une slow science critique. Il serait temps de nous en rendre compte et de nous mobiliser à dessein si nous ne voulons pas que nos universités se transforment en "super lycées" où les enseignements faisant fond sur la recherche seront remplacés par des cours «clicbouton» et les collectifs de recherche troqués pour des structures d'opportunité pour carriéristes aux dents longues qui vivent leurs «avancements » comme la conséquence évidente de leurs propriétés individuelles et non comme un effet des positions qu'ils ont pu ou su occuper - de fait, le sens du placement demande parfois un certain talent.

\section{NOTES}

1. «On a l'impression que les délinquants et criminels ont lu les travaux de la sociologie et ont trouvé une bonne raison de commettre leur crime parce qu'ils se sentiraient déresponsabilisés ! On croit rêver. Qu'un acteur politique prononce de telles âneries, on finit presque par s'habituer, mais quand des sociologues disent cela c'est proprement ahurissant. Ces gens pensent sans doute qu'en supprimant les chercheurs qui mettent au jour des inégalités ou des déterminations sociales bien réelles on pourra enfin vivre dans un monde libre et égalitaire. Ils marchent sur la tête. La sociologie décrit et interprète le monde, et quand elle observe des déterminations sociales, des régularités, des logiques sociales elle les porte aux yeux de tous ceux et celles qui veulent bien les lire. Mais je ne vois pas quels effets performatifs elle pourrait bien jouer sur les tendances de fond du monde social qui n'attendent pas les sociologues pour continuer à œuvrer et façonner le monde social. Et d'ajouter : [...] La sociologie souffre surtout de ne pas être suffisamment lue et diffusée, et non des effets "catastrophiques" qu'on lui prête. Sur quelles enquêtes s'appuient ceux qui parlent de ses effets ? Où et par quels moyens ont-ils vus ces effets jouer sur les comportements de nos concitoyens ? Je serai intéressé de connaitre les résultats de ces travaux» (https://www.franceculture.fr/sociologie/sept-pechescapitaux-sociologie-vieille-histoire). 
INDEX

Mots-clés : sociologie critique, sociologie analytique, fachosphère, sciences de l'information et de la communication, Bernard Lahire

\section{AUTEUR}

\section{FABIEN GRANJON}

Professeur des Universités, Paris 8 